\title{
Development of Improved Mumps Vaccine Candidates by Mutating Viral mRNA Cap Methyltransferase Sites in the Large Polymerase Protein
}

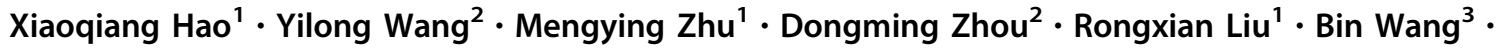 \\ Yao-Wei Huang ${ }^{3}$ (1) $\cdot$ Zhengyan Zhao $^{1,2}$ (1)
}

Received: 26 July 2020 / Accepted: 20 October 2020 / Published online: 7 December 2020

(C) Wuhan Institute of Virology, CAS 2020

\begin{abstract}
Although a live attenuated vaccine is available for controlling mumps virus $(\mathrm{MuV})$, mumps still outbreaks frequently worldwide. The attenuated MuV vaccine strain S79 is widely used in mumps vaccination in China, but still with many shortcomings, among which the most prominent are the side effects and decreased immunity. Therefore, there is a need to further improve the safety and efficacy of the current MuV vaccine. In the present study, we further attenuated MuV S79 vaccine strain by inhibiting viral mRNA methyltransferase (MTase). We generated a panel of eight recombinant MuVs $(\mathrm{rMuVs})$ carrying mutations in the MTase catalytic site or S-adenosylmethionine (SAM) binding site in the large (L) polymerase protein. These rMuVs are genetically stable and seven rMuVs are more attenuated in replication in cell culture and five rMuVs are more attenuated in replication in lungs of cotton rats compared with the parental vaccine strain S79. Importantly, cotton rats vaccinated with these seven $\mathrm{rMuV}$ mutants produced high levels of serum neutralizing antibodies and were completely protected against challenge with a wild-type $\mathrm{MuV}$ strain (genotype F). Therefore, our results demonstrate that alteration in the MTase catalytic site or SAM binding site in MuV L protein improves the safety or the immunogenicity of the MuV vaccine and thus mRNA cap MTase may be an effective target for the development of new vaccine candidates for $\mathrm{MuV}$.
\end{abstract}

Keywords Mumps virus $(\mathrm{MuV}) \cdot$ Vaccine $\cdot$ Methyltransferase (MTase) $\cdot$ Large polymerase protein

\section{Introduction}

Xiaoqiang Hao and Yilong Wang have contributed equally to this work.

Electronic supplementary material The online version of this article (https://doi.org/10.1007/s12250-020-00326-y) contains supplementary material, which is available to authorized users.

\footnotetext{
Zhengyan Zhao

Zhaozy@zju.edu.cn

Yao-Wei Huang

yhuang@zju.edu.cn

1 Zhejiang University School of Medicine, Hangzhou 310058, China

2 The Children's Hospital, Zhejiang University School of Medicine, Hangzhou 310052, China

3 Institute of Preventive Veterinary Medicine and Key Laboratory of Animal Virology of Ministry of Agriculture, Department of Veterinary Medicine, Zhejiang University, Hangzhou 310058, China
}

Mumps is a common respiratory infection in children caused by mumps virus (MuV) (Muhlemann 2004). The primary symptoms of $\mathrm{MuV}$ infection are fever and swelling of the parotid gland and can also cause complications including orchitis, deafness, aseptic meningitis and encephalitis (Galazka et al. 1999). Since 1967, live attenuated vaccines have been developed for immunization against mumps in children, which significantly reduced the health, social and economic problems caused by mumps (Buynak and Hilleman 1966). Currently, live attenuated mumps vaccines widely used in the world include JerylLynn, S79, Urabe Am9, RIT 4385, Leningrad-3, Leningrad-Zagreb, Rubini, etc. (Rubin et al. 2015). Live attenuated $\mathrm{S} 79 \mathrm{MuV}$ vaccine which is originated from Jeryl Lynn strain has been licensed for vaccination since 1996 in China (Wu et al. 2018). Although live attenuated MuV vaccine is efficacious, it can rarely cause some undesirable 
adverse effects (Di Pietrantonj et al. 2020). In fact, many vaccinated children may experience side effects including fever, rash, lymphadenopathy, parotitis. In addition, the incidence of mumps is still high after a dose of measlesmumps-rubella (MMR) combination vaccine introduced in China (Cui et al. 2018; Ma C et al. 2018; Wu et al. 2018). Even vaccination coverage of the later second dose is high, it does not effectively prevent outbreaks of mumps among adolescents worldwide (Beleni and Borgmann 2018; Lewnard and Grad 2018; Ma R et al. 2018; Fields et al. 2019; Westphal et al. 2019). To solve the recurrence of mumps outbreaks, a proposal for a third dose of MMR vaccine against young adults has been proposed (Albertson et al. 2016; Marin et al. 2018). However, the third dose of $\mathrm{MuV}$ vaccine can control but not prevent outbreaks (Cardemil et al. 2017). Therefore, there is still an urgent need to develop more effective and safer $\mathrm{MuV}$ vaccines (May et al. 2018).

$\mathrm{MuV}$ is an enveloped, non-segmented negative-sense (NNS) RNA virus, belonging to the genus Orthorubulavirus, subfamily Rubulavirinae in the family Paramyxoviridae. The MuV genome encoded 7 transcription units: the nucleo $(N), \mathrm{V} /$ phospho/I $(V / P / I)$, matrix $(M)$, fusion $(F)$, small hydrophobic $(S H)$, hemagglutinin-neuraminidase $(H N)$ and large $(L)$ protein genes (Elango et al. 1988; Elliott et al. 1990).

The traditional method for developing live attenuated mumps vaccine is through continuous low-temperature passages in chicken embryo cells to identify an optimized viral strain with reduced virulence but sufficient immunogenicity (Buynak and Hilleman 1966). In addition, Japanese researchers developed several temperaturestable mutants among clinically isolated viral strains by nitrosoguanidine and ultraviolet light (Saika et al. 2006). However, these strategies are complicated and timeconsuming and the resultant mutants are often unstable. The reverse genetics system provides a rapid and efficient strategy for rational design of live attenuated vaccines (Bukreyev et al. 1996; Xu et al. 2014; Nogales and Martinez-Sobrido 2016; Yang et al. 2016). The first MuV reverse genetics system was reported in 2000 (Clarke et al. 2000). As with other NNS RNA viruses (Garcin et al. 1995; Lawson et al. 1995; Jin et al. 1998), co-transfection of plasmids expressing viral anti-genome and support plasmids encoding viral nucleocapsid complex $(\mathrm{N}, \mathrm{P}$ and $\mathrm{L}$ protein) was essential for the recovery of MuV. Using reverse genetics, researchers have shown that deletion of $\mathrm{SH}$ gene from $\mathrm{MuV}$ genome can further weaken the $\mathrm{MuV}$ vaccine strains and increase their immunogenicity. However, follow-up studies showed that this method might not be applicable to all vaccine strains (Xu et al. 2011). Therefore, exploration of new strategy to further improve the safety and efficacy is still needed.
The large (L) protein of NNS RNA viruses is the catalytic subunit of RNA dependent RNA polymerase that carries out replication and transcription (Whelan et al. 2004). During transcription, NNS RNA viruses produce mRNAs that are capped and methylated at $5^{\prime}$-end and polyadenylated at $3^{\prime}$-end (Poch et al. 1990). The mRNA capping and methylation are catalyzed by the $\mathrm{L}$ protein. Specifically, addition of mRNA cap is accomplished by the conserved $\mathrm{V}$ (CR V) of $\mathrm{L}$ protein. The mRNA cap structure is further methylated by guanine-N-7 (G-N-7) and ribose 2'-O methyltransferases (MTases) which are located in the CR VI of L protein ( $\mathrm{Li}$ et al. 2006). Recently, cryo-EM structure of $L$ proteins of two NNS RNA viruses, vesicular stomatitis virus (VSV) and human respiratory syncytial virus (RSV), has been solved (Ge et al. 2010; Gilman et al. 2019).

mRNA cap G-N-7 methylation is essential for viral protein translation (Muthukrishnan et al. 1976) whereas ribose $2^{\prime}-\mathrm{O}$ methylation is associated with viral evasion of host interferon (IFN)-mediated innate immune responses (Zust et al. 2011). Therefore, mRNA cap methylation is an attractive target for development of antiviral drugs and live attenuated vaccines. Because virus lacking mRNA cap G-N-7 methylation will reduce viral protein translation, which in turn down-regulate replication and transcription, leading to virus attenuation. Viruses lacking mRNA cap ribose $2^{\prime}-\mathrm{O}$ methylation will make the virus more easily recognized by host cells, activate the interferon system and then promote $\mathrm{T}$ cell immune response and $\mathrm{B}$ cell immune response and improve immunogenicity. This finding has been proven in several NNS RNA viruses, including measles virus (MV), human metapneumovirus (hMPV), avian metapneumovirus (aMPV), rabies virus (RABV) and vesicular stomatitis virus (VSV) (Ma et al. 2014; Sun et al. 2014; Zhang et al. 2014; Wang et al. 2018). These studies showed that recombinant viruses lacking MTase activity were highly attenuated both in vitro and in vivo yet retained high immunogenicity.

We hypothesized that reducing MTase activity can further improve the safety and immunogenicity of mumps vaccines. Using the robust reverse genetics system of Chinese S79 vaccine strain (MuV-S79) established in our previous research (Zhou et al. 2019b), we generated a total of eight recombinant MuVs with amino acid substitutions in the MTase domain of the $\mathrm{L}$ protein. These rMuVs mutant strains were significantly more attenuated in cell culture compared with the parental vaccine strain and were genetically stable when passing repeatedly in cell culture for 10 passages. In addition, we found that three $\mathrm{rMuV}$ mutants were significantly more attenuated in cell culture and in cotton rats yet retained wild-type level of immunogenicity. We also identified one $\mathrm{rMuV}$ mutant that was replicated as efficiently as the parental vaccine strain 
but had a higher immunogenicity. Collectively, our results suggest that $\mathrm{rMuV}$ carrying mutations in the SAM binding site or MTase catalytic site may have improved safety and/ or immunogenicity.

\section{Materials and Methods}

\section{Cells and Viruses}

BHK-SR19-T7 cells (kindly offered by Apath, LLC, Brooklyn, NY), Vero cells (African green monkey kidney, ATCC-CCL-81) and Hela cells (kindly offered by Wangxing Hu, Ph.D., The Second Affiliated Hospital of Zhejiang University School of Medicine) were grown in Dulbecco's modified Eagle's medium (DMEM; Life Technologies) supplemented with $10 \%$ fetal bovine serum (FBS, Life Technologies, USA). To screen BHK-SR19-T7 cells expressing $\mathrm{T} 7$ polymerase, the medium of the cells was supplemented with $10 \mu \mathrm{g} / \mathrm{mL}$ puromycin (Life Technologies) during passages.

The MuV-S79 vaccine strain (kindly provided by Professor Yiyu Lu, Zhejiang CDC, China) and MuV wild-type strain (isolated from clinical samples in Children's Hospital, Zhejiang University School of Medicine, genotype F) were passaged in Vero cells. Recombinant rMuVS79-A1814G, rMuV-S79-G1816A, rMuV-S79-G1818A, rMuV-S79-D1892A, were mutated from the methyltransferase binding site of rMuV-S79. And rMuV-S79-K1792A, rMuV-S79-D1917A, rMuV-S79-K1953A, rMuV-S79E1990A, were mutated from the methyltransferase catalytic site of rMuV-S79. Viruses were handled in BSL-2 laboratory adhered to standard biosecurity and institutional safety procedures.

\section{Specimen and Virus Separation}

23 clinical samples of mumps-positive throat swabs were used to isolate and identify the wild type virus. RT-PCR tests were performed on clinical mumps throat swab samples. $100 \mu \mathrm{L}$ of positive clinical mumps samples were inoculated into Vero cell monolayers and adsorbed at $37^{\circ} \mathrm{C}$ for $1 \mathrm{~h}$. The Vero cells were discarded the adsorbed liquid and added $2 \mathrm{~mL}$ of DMEM medium containing $2 \%$ FBS and cultured at $37^{\circ} \mathrm{C}$. Cytopathic effects (CPE) were observed and recorded daily. If no CPE was observed, the supernatant was harvested 7 days after incubation and blindly passaged three times on a new Vero cell monolayer. When significant CPE was observed, the cells were frozen and thawed three times to harvest the viral supernatant and stored at $-80{ }^{\circ} \mathrm{C}$. The isolated virus was plaque purified and confirmed by RT-PCR and used as the MuV wild-type strain in the study.

\section{Site-directed Mutagenesis}

rMuVs mutants were obtained by specific amino acid mutations in the CR VI of rMuV-S79 L protein. Using the PFU Site-Directed Mutagenesis Kit (NEB) and using rMuV-S79 (GenBank: MT732483) as a template, the key amino acids (K1792, G1816, G1818, D1892, D1917, K1953, E1990) were mutated to alanine by primers (Supplementary Table S1). And A1814 was mutated from alanine to glycine. The mutation was confirmed by sequencing. These plasmids were named pYESMuV-S79-K1792A, pYES-MuV-S79-A1814G, pYES-MuVS79-G1816A, pYES-MuV-S79-G1818A, pYES-MuV-S79D1892A, pYES-MuV-S79-D1917A, pYES-MuV-S79K1953A and pYES-MuV-S79-E1990A.

\section{Recovery of Infectious rMuV-S79 from Transfected Cells}

The full-length MuV clone pYES-MuV-S79 $(12.5 \mu \mathrm{g})$ and its supporting plasmids pT7-MuV-S79-NP $(3.75 \mu \mathrm{g})$, pT7MuV-S79-P $(3.75 \mu \mathrm{g})$, pT7-MuV-S79-L $(1.25 \mu \mathrm{g})$ were co-transfected into 70\%-80\% confluent BHK-SR-19-T7 cells in T25 cell culture flask. The transfection was operated by using Lipofectamine 2000 and referring to the manufacturer's instructions. After $6 \mathrm{~h}$ of incubation at $37{ }^{\circ} \mathrm{C}$, fresh Opti-MEM culture was added to replace the old medium. At $72 \mathrm{~h}$ post-transfection, transfected cells were scraped off, resuspended and co-cultured with $75 \%$ confluent Vero cells in T25 cell culture flask at $37{ }^{\circ} \mathrm{C}$ with $5 \% \mathrm{CO}_{2}$ for 3-7 days until mumps-induced specific cytopathic effect was observed. Each recombinant virus was plaque isolated.

\section{Immunofluorescence Assay}

24-well Vero cells $\left(5 \times 10^{4} /\right.$ well $)$ were grown overnight for being infected with rMuVs. After $1 \mathrm{~h}$ of adsorption, the virus adsorption solution was discarded and the cells were washed twice with PBS and added with $0.5 \mathrm{~mL}$ DMEM containing 2\% FBS. After $48 \mathrm{~h}$, cells were washed twice with PBS and fixed with $4 \%$ paraformaldehyde in PBS for $30 \mathrm{~min}$ at room temperature (RT), then ruptured with $0.2 \%$ Triton X-100 in PBS for 20 min and blocked with $2 \%$ BSA in PBS for $1 \mathrm{~h}$. Further, the treated cells and the specific mouse anti-mumps $N$ protein specific antibody (ab9876, Abcam, 1:20) were incubated for $1 \mathrm{~h}$ at room temperature, following incubation with anti-mouse IgG (A21203, Alexa Fluor ${ }^{\circledR} 594$ donkey, Invitrogen $\left.1: 2000\right)$ for $0.5 \mathrm{~h}$ at RT. Finally, the nucleus was stained by DAPI $(1: 10,000)$ and incubated for $5 \mathrm{~min}$ in the dark; and the results were observed under a fluorescence microscope. 


\section{Western Blot}

After discarding the medium and washing the cells with PBS, Vero cells in 6-well plates were inoculated with $\mathrm{rMuVs}$ at a multiplicity of infection (MOI) of 0.1 . The virus was collected per $24 \mathrm{~h}$ by freezing and thawing the cells three times and centrifuged at $4000 \mathrm{~g}, 4{ }^{\circ} \mathrm{C}$ for $25 \mathrm{~min}$. The cell lysate was denatured at $100{ }^{\circ} \mathrm{C}$ for $5 \mathrm{~min}$ and analyzed on a $10 \%$ polyacrylamide bis-Tris gel. The separated protein was transferred to a polyvinylidene difluoride membrane. The membranes were blocked with 5\% skim milk in PBS and subsequently incubated with specific mouse anti-mumps $\mathrm{N}$ protein specific antibody (ab9876, Abcam, 1:100) for $1 \mathrm{~h}$ at RT, following incubation with anti-mouse $\operatorname{IgG}(7076 \mathrm{~S}, \mathrm{CST}, 1: 3000)$ for $0.5 \mathrm{~h}$ at RT. Finally, the membranes and ECL were incubated (20-50-120, Biological Industries) for $2 \mathrm{~min}$ and then developed.

\section{Virus Titration}

Prepare $100 \mathrm{~mL}$ of the covering medium one day in advance, components: $3.2 \%$ aqueous solution of sodium carboxymethylcellulose $25 \mathrm{~mL}$ (autoclave for $30 \mathrm{~min}$ ), $75 \mathrm{~mL}$ DMEM medium containing 2\% FBS and 1\% PS. Discard the medium of Vero cells in six-well plates at a density of $5 \times 10^{5}$ cells per well and wash the cells with PBS before adsorption of serial dilutions of mumps. After shaking in constant temperature for $1 \mathrm{~h}$, virus adsorption solution was discarded and cells were covered with $2 \mathrm{~mL}$ of the covering medium per well. Then 6-well plates were placed at $37{ }^{\circ} \mathrm{C}, 5 \% \mathrm{CO}_{2}$ cell incubator for 7 days. After 7 days, cells were fixed $2 \mathrm{~mL}$ of $4 \%$ formaldehyde for $2 \mathrm{~h}$ per well. The plaques formed by the mumps virus infection were visualized by staining with $0.05 \%$ (wt/vol) crystal violet each well for $15 \mathrm{~min}$. Calculate the virus titration by counting the number of plaques in the cell and combining the dilution gradient.

\section{Virus Plaques Size}

The medium of Vero cells was discarded and the cells were washed with PBS. Vero cells were adsorbed 100 PFU of each virus per well. After shaking in constant temperature for $1 \mathrm{~h}$, virus adsorption solution was discarded and cells were covered with $2 \mathrm{~mL}$ of the covering medium per well. Then 6-well plates were placed in cell incubator at $37{ }^{\circ} \mathrm{C}$ with $5 \% \mathrm{CO}_{2}$ for 7 days. After 7 days, cells were fixed with $2 \mathrm{~mL}$ of $4 \%$ formaldehyde for $2 \mathrm{~h}$ per well. The plaques formed by the mumps virus infection were visualized by staining with $0.05 \%$ (wt/vol) crystal violet each well for $15 \mathrm{~min}$. The bottoms of the six-well plate were scanned after crystal violet staining and the diameter of the virus plaque was measure by the 'ruler' tool in the PS software. 10-15 plaques were randomly picked to measure their diameter and averaged as the final size of the plaque.

\section{Recombinant Virus Replication Kinetics in vitro}

After discarding the medium and washing the cells with PBS, Vero/Hela cells in 6-well plates were inoculated with rMuVs at a multiplicity of infection (MOI) of 0.1. The virus was collected per $24 \mathrm{~h}$ by freezing and thawing the cells three times and centrifuged at $4000 \mathrm{~g}, 4{ }^{\circ} \mathrm{C}$ for $25 \mathrm{~min}$. Virus titers were determined by plaque assays as described above (Virus titration). The experiment was repeated for 3 times to plot growth curves.

\section{Genetic Stability of rMuV-S79 Mutants in vitro}

Inoculation of Vero cells in T25 flasks with mRNA methyltransferase-deficient mumps virus vaccine strain or $\mathrm{rMuV}-\mathrm{S} 79$ at $\mathrm{MOI}=0.1$. After virus infection of Vero cells for $1 \mathrm{~h}$, the virus adsorption solution was discarded, then the cells were washed with PBS and $4 \mathrm{~mL}$ of DMEM medium containing $2 \%$ FBS was added to each well and cells were placed in a $37{ }^{\circ} \mathrm{C}$ cell culture incubator. After CPE reaches $80 \%$ of the cell monolayer, the virus was collected by freezing and thawing the cells three times and centrifuged at $4000 \mathrm{~g}, 4{ }^{\circ} \mathrm{C}$ for $25 \mathrm{~min}$. The collected virus was used for the next passage. Repeat the passage 10 times as described above. Inoculation of primary chicken embryo fibroblast cells (CEF) in T25 flasks with mRNA methyltransferase-deficient mumps virus vaccine strain or $\mathrm{rMuV}$ $\mathrm{S} 79$ at $\mathrm{MOI}=1$. After $72 \mathrm{~h}$, the virus was collected by freezing and thawing the cells three times and centrifuged at $4000 \mathrm{~g}, 4{ }^{\circ} \mathrm{C}$ for $25 \mathrm{~min}$. The collected virus was used for the next passage. Repeat the passage 3 times as described above.

The entire genome of each recombinant virus was amplified by RT-PCR and sequenced. The genomic information of each methyltransferase-deficient $\mathrm{rMuVs}$ was amplified by RT-PCR and sequenced.

\section{Confirmation of Sequences by RT-PCR}

Viral RNA was extracted by using RNeasy mini-kit (Qiagen) according to the manufacturer's instructions. A $950 \mathrm{bp}$ DNA fragment of CR VI derived from MuV L protein was amplified by a One-Step RT-PCR kit (Qiagen) according to the manufacturer's instructions using primers MuV-13722F and MuV-14671R (Supplementary Table S1). To confirm that the designed mutation was indeed present in the CR VI of the MuV L protein, the PCR product was sequenced using the primers described above. 


\section{Purification of rMuV-S79}

Ten T150 flasks of more than $90 \%$ confluent Vero cells were adsorbed with mRNA methyltransferase-deficient mumps virus with $\mathrm{MOI}=0.1$. After $80 \%$ of cytopathic effects (CPE) were observed, the virus was collected by freezing and thawing the cells three times and centrifuged at $4000 \mathrm{~g}, 4{ }^{\circ} \mathrm{C}$ for $25 \mathrm{~min}$. Then the virus was pelleted by ultracentrifugation at $25,000 \mathrm{~g}$ for $2 \mathrm{~h}$ and resuspend in $500 \mu \mathrm{L}$ of DMEM containing $10 \%$ Trehalose. The ultracentrifuge tube was placed on ice overnight to completely dissolve the suspended virus. Purified virus was aliquoted and stored at $-80{ }^{\circ} \mathrm{C}$ for subsequent animal testing the next day.

\section{Animal Experiments}

Female specific-pathogen-free (SPF) cotton rats (kindly provided by Professor Enmei Liu from Children's hospital of Chongqing Medical University), 4-5 weeks old, were used in the experiment. The experimental animals were housed within the facilities of the Children's Hospital of Chongqing Medical University. During the experiment, cotton rats were anesthetized using isoflurane and sacrificed by carbon dioxide asphyxiation.

\section{Replication of rMuV-S79s in Cotton Rats}

In this animal experiment, the virulence assay of mRNA methyltransferase-deficient mumps virus vaccine strain was performed. Forty-five 4 weeks old, female SPF cotton rats were randomly divided into 9 groups: rMuV-S79, rMuV-S79-K1792A, rMuV-S79-G1816A, rMuV-S79G1818A, rMuV-S79-D1892A, rMuV-S79-D1917A, rMuVS79-K19538A, rMuV-S79-E1990A and DMEM. Isoflurane anesthetized cotton rats were inoculated intranasally at a dose of $1.0 \times 10^{6} \mathrm{PFU}$. After inoculation, clinical signs, weight and mortality were observed and recorded accordingly. On the 4th day after inoculation, cotton rats were euthanized and lung, spleen and brain tissue samples were collected. The collected left lungs, left brain and spleen tissues were weighed and then added to $1 \mathrm{~mL}$ of pre-cooled DMEM for grinding and homogenization, then centrifuged at $4000 \mathrm{~g}$ for $4 \mathrm{~min}$ at $4{ }^{\circ} \mathrm{C}$. The supernatant was collected and stored in a new pre-cooled sterile tube for plaque assay. For histopathological detection, the right lung and right brain tissues were fixed in $4 \%$ formaldehyde. Fixed tissues were embedded in paraffin and sectioned at $5 \mu \mathrm{m}$. Slides were then stained with hematoxylin-eosin (H\&E) for the examination of histological changes by light microscopy.
Immunogenicity of rMuV-S79s in Cotton Rats

In this animal experiment, the immunity assay of constructed vaccine strains was performed on forty-five 4-week-old female SPF cotton rats by same grouping method as above. Isoflurane anesthetized cotton rats were inoculated intranasally at a dose of $1.0 \times 10^{6} \mathrm{PFU} \mathrm{rMuVs}$ and the serum of the cotton rat was collected at the 2 nd, 3rd, 4th, 5th, 7th and 9th week after inoculation for antibody detection. The animals were challenged with wildtype $\mathrm{MuV}$ strain by intranasal inoculation with a dose of $1.0 \times 10^{7} \mathrm{PFU} / 100 \mu \mathrm{L}$ and then the morbidity and mortality of cotton rats were observed daily. Four days after challenge, all cotton rats were euthanized and lung tissues were collected for detection of intrapulmonary virus titers and histopathological research as described above.

\section{Immunohistochemical (IHC) Staining}

First, the tissue samples were fixed with $4 \%$ formaldehyde and embedded in paraffin. The slices were cut into $4 \mu \mathrm{m}$ thick slices and then incubated at $60^{\circ} \mathrm{C}$ for $2 \mathrm{~h}$. Subsequently, the slides were de-waxed and then put into a beaker filled with repair liquid and then put in a pressure cooker to boil. After heating at the set pressure for $2 \mathrm{~min}$, the slides were cooled by water. Then The sections were incubated in $3 \% \mathrm{H}_{2} \mathrm{O}_{2}$ solution about $10 \mathrm{~min}$ to block endogenous peroxidase activity. The slides were incubated with normal goat serum ( $1 / 20$ dilution in PBS) at $37{ }^{\circ} \mathrm{C}$ for 15 min and subsequently with mouse anti-mumps $\mathrm{N}$ protein mAb (Abcam, ab9880, 1/500 dilution in PBS) at $4{ }^{\circ} \mathrm{C}$ overnight. Afterward, the slides were cultivated with a biotinylated goat anti-mouse HRP-IgG (ZSGB-BIO, PV$8000,1 / 1)$ at $37^{\circ} \mathrm{C}$ for $30 \mathrm{~min}$. The slides were then developed using a diaminobenzidine (DAB) for $2 \mathrm{~min}$ and hematoxylin for $20 \mathrm{~s}$ as a counterstain.

\section{Detection of Mumps Virus Neutralizing Antibody Levels}

Mumps virus-specific neutralizing antibody titers in cotton rat serum were detected by plaque reduction neutralization assay. The collected serum samples were inactivated in a constant temperature water bath at $56{ }^{\circ} \mathrm{C}$ for $30 \mathrm{~min}$. Then the inactivated serum samples were serially diluted with DMEM medium in 96-well plates (1:16 to 1:2048) mixed with an equal volume of DMEM containing approximately $100 \mathrm{PFU} /$ well rMuV-S79 and incubated for $1 \mathrm{~h}$ at $37{ }^{\circ} \mathrm{C}$ constant temperature shaker. The virus-serum mixture was adsorbed to single-layer Vero cells in 6-well plates. Further virus adsorption solution was discarded and cells were covered with $2 \mathrm{~mL}$ of the covering medium (as virus titration) per well, then 6-well plates were placed in cell 


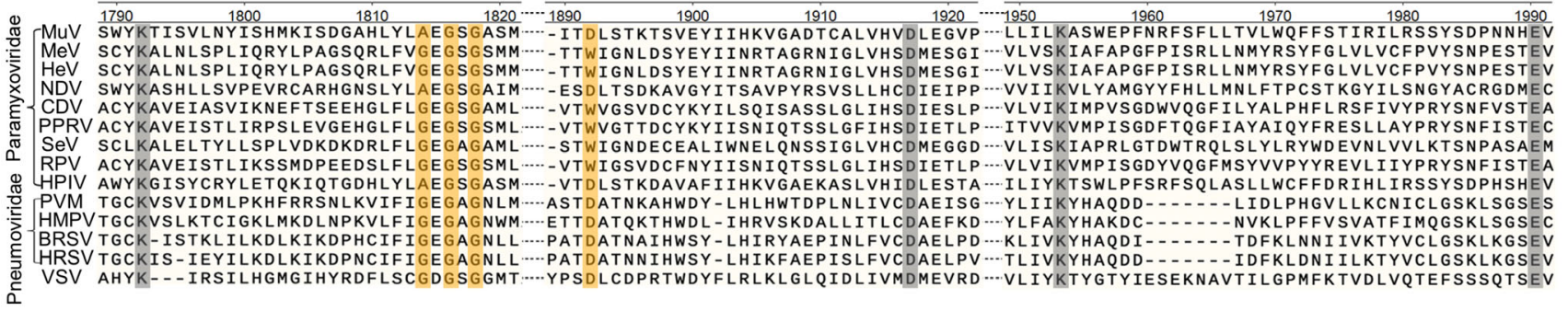

Fig. 1 Sequence alignment in the conserved region VI (CR VI) of the $\mathrm{L}$ proteins of NNS RNA viruses. The sequence of CR VI in the $L$ protein was selected and compared using the Clustal Omega software. The coordinates were based on the amino acids 1790-1990 of the $\mathrm{L}$ protein of $\mathrm{MuV}-\mathrm{S} 79$. The predicted SAM binding sites (GxGxG...D) are shown in orange and methyltransferase catalytic sites (K-D-K-E) are shown in grey. Representative members in the Paramyxoviridae (MuV, mumps virus; $\mathrm{MeV}$, Measles virus; $\mathrm{HeV}$,

incubator at $37{ }^{\circ} \mathrm{C}$ with $5 \% \mathrm{CO}_{2}$ for 7 days. After 7 days, cells were fixed with $2 \mathrm{~mL}$ of $4 \%$ formaldehyde per well for $2 \mathrm{~h}$. The plaques formed by the mumps virus infection were visualized by staining with $0.05 \%$ (wt/vol) crystal violet for $15 \mathrm{~min}$. The plaques were counted and $50 \%$ plaque reduction titers were calculated as the $\mathrm{MuV}$-specific neutralizing antibody titers.

\section{Sequence Data of rMuVs and Wild Type MuV}

Sequence data of rMuVs that support the findings of this study have been deposited in GenBank with the accession numbers: rMuV-S79, MT732483; rMuV-S79-K1792A, MT732484; rMuV-S79-A1814G, MT732485; rMuV-S79G1816A, MT732486; rMuV-S79-G1818A, MT732487; rMuV-S79-D1892A, MT732488; rMuV-S79-D1917A, MT732489; MuV-S79-K1953A, MT732490; rMuV-S79E1990A, MT732491. The wild type mumps virus GenBank accession number: KF170916.

\section{Statistical Analysis}

Statistical analysis of data was performed using two-way ANOVA of Prism, version 8.0. $P$ values $(P)$ Less than 0.05 were considered statistically significant. $* P<0.05$, $* * P<0.01, * * * P<0.001, * * * * P<0.0001$.

\section{Results}

\section{Design of Amino Acid Substitutions in the MTase Catalytic Site and SAM Binding Site of MuV L Protein}

We first performed a sequence alignment of the CR VI (MTase region) of the L proteins among NNS RNA viruses
Hendra virus; NDV, Newcastle disease virus; CDV, canine distemper virus; PPRV, peste des petits ruminants virus; $\mathrm{SeV}$, Sendai virus; RPV, Rinderpest virus; HPIV, human parainfluenza virus-2), Pneumoviridae (PVM, pneumonia virus of mice; HMPV, human metapneumovirus; BRSV, bovine respiratory syncytial virus; HRSV, human respiratory syncytial virus), Rhabdoviridae (VSV, vesicular stomatitis virus) were selected for sequence alignment.

(Fig. 1). An MTase catalytic site (K-D-K-E tetrad) is conserved in the CR VI of all $\mathrm{L}$ proteins. In the methylation reaction, the MTase must bind to the methyl donor, SAM, in order to achieve methylation. A SAM binding site (GxGxG...D motif) is conserved in the L proteins of NNS RNA viruses. However, the SAM binding site of the L protein of MuV, Human parainfluenza virus-2 (HPIV2) and Newcastle disease virus (NDV) contains a naturally occurring mutation. Specifically, the first amino acid residue in the SAM biding site is an alanine but not a glycine. Sequence alignment indicated that amino acids including A1814, G1816, G1818 and D1892 responsible for SAM binding in $\mathrm{MuV} L$ protein; and amino acids K1792, D1917, K1953 and E1990 correspond to the catalytic K-D-K-E tetrads of the $\mathrm{MuV} \mathrm{L}$ protein. We performed an alanine scanning mutagenesis in the SAM binding site and K-D-K-E motif in MuV L protein and each of these amino acid residues was mutated to alanine. Since the first amino acid of the SAM binding site is naturally an alanine (A1814), we mutated this alanine to glycine to create a SAM binding site which is conserved in the L proteins of most NNS RNA viruses. Primers used for mutagenesis are listed in Supplementary Table S1. Each of these mutations was introduced into plasmid pYES-MuV-S79 encoding a full-length cDNA clone of MuV vaccine strain S79.

\section{Recovery of rMuVs Carrying Mutations in the MTase Region of $L$ Protein}

Using the reverse genetics system, all eight $\mathrm{rMuVs}$ were recovered, which were named $\mathrm{rMuV}-\mathrm{S} 79-\mathrm{K} 1792 \mathrm{~A}, \mathrm{rMuV}$ S79-A1814G, rMuV-S79-G1816A, rMuV-S79-G1818A, rMuV-S79-D1892A, rMuV-S79-D1917A, rMuV-S79K1953A and rMuV-S79-E1990A. MuV-induced CPE were observed after 2-4 days (Fig. 2A). Subsequently, MuV NP protein was detected in Vero cells infected by each 


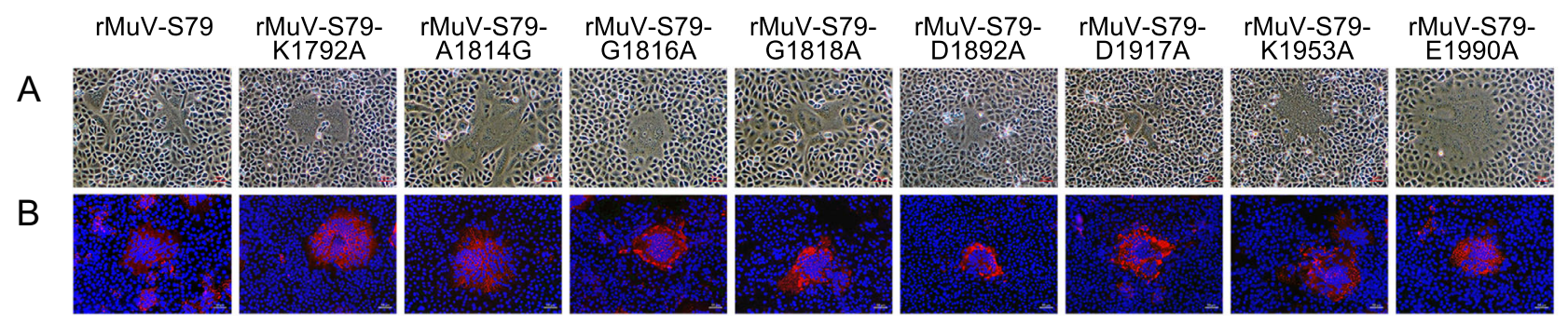

Fig. 2 Rescue recombinant $\mathrm{MuV}$ mutants using reverse genetics. BHK-SR-19-T7 cells were co-transfected with a plasmid encoding full-length $\mathrm{MuV}$ antigenome and supporting plasmids encoding $N$, $P$ and $L$ genes. At day 3 post-transfection, cells were harvested and co-cultured with Vero cells for 24 to $96 \mathrm{~h}$. Cell culture supernatants

recombinant virus using an immunofluorescence assay (IFA) and Western blot (Fig. 2B and Supplementary Fig. S1A). The parent strain obtained the top NP antigen protein expression at $72 \mathrm{~h}$ after transfection, while rMuVs were delayed by 1-3 days (Supplementary Fig. S1B). The top NP antigen protein expression of rMuV-S79-D1917A and $\mathrm{rMuV}-\mathrm{S} 79-\mathrm{E} 1990 \mathrm{~A}$ are similar to the parent virus, while the top expression of other $\mathrm{rMuVs}$ is lower than the parent virus (Supplementary Fig. S1C). The sizes of virusinduced plaques differed between the rescued parental and mutant viruses (Supplementary Fig. S2). Each recombinant virus was plaque purified, as demonstrated in Fig. 3 and $4 \mathrm{~A}, \mathrm{rMuV}-\mathrm{S} 79-\mathrm{K} 1792 \mathrm{~A}(0.64 \pm 0.11 \mathrm{~mm})$, rMuVS79-G1816A $\quad(0.66 \pm 0.10 \mathrm{~mm}), \quad$ rMuV-S79-G1818A $(0.52 \pm 0.13 \mathrm{~mm}), \mathrm{rMuV}-\mathrm{S} 79-\mathrm{D} 1892 \mathrm{~A}(0.18 \pm 0.09 \mathrm{~mm})$ and $\mathrm{rMuV}-\mathrm{S} 79-\mathrm{D} 1917 \mathrm{~A}(0.48 \pm 0.10 \mathrm{~mm})$ formed significantly smaller plaques in diameter compared to the parental rMuV-S79 $(0.82 \pm 0.16 \mathrm{~mm})$. Interestingly, $\mathrm{rMuV}-\mathrm{S} 79-\mathrm{A} 1814 \mathrm{G} \quad(1.23 \pm 0.18 \mathrm{~mm})$ formed bigger were used for next passage in Vero cells. A Specific cytopathic effects of rescued mumps virus on Vero cells. Scale bar: $50 \mu \mathrm{m}$. B Indirect immunofluorescence analysis of MuV rescued by Vero cells at $48 \mathrm{~h}$ post infection, red is labeled as mumps $\mathrm{N}$ protein and blue is labeled as Vero cell nucleus. Scale bar: $50 \mu \mathrm{m}$.

plaques than the parental virus. Recombinant rMuV-S79K1953A $(0.85 \pm 0.12 \mathrm{~mm})$ and rMuV-S79-E1990A $(0.70 \pm 0.13 \mathrm{~mm})$ had similar plaque sizes with the parental rMuV-S79 strain. Finally, the genome of each recombinant virus was amplified by RT-PCR and sequenced (Supplementary Fig. S3). The results showed that each $\mathrm{rMuV}$ contained only the designed mutation.

\section{Replication Kinetics of rMuV-S79s}

We evaluated the replication kinetics of these recombinant MuV mutants in Vero cells (Fig. 4B). The virus growth of most rMuV-S79 mutants was delayed by 1 day compared with the parental rMuV-S79. However, rMuV-S79A1814G replicated earlier than the parental rMuV-S79 and reached a peak titer $6.75 \pm 0.26 \log _{10} \mathrm{PFU} / \mathrm{mL}$. The peak titers of rMuV-S79-K1792A and rMuV-S79-D1892A were $5.7 \pm 0.59 \log _{10} \mathrm{PFU} / \mathrm{mL}$ and $6.0 \pm 0.89 \log _{10} \mathrm{PFU} / \mathrm{mL}$, respectively, which were lower than that of $\mathrm{rMuV}-\mathrm{S} 79$

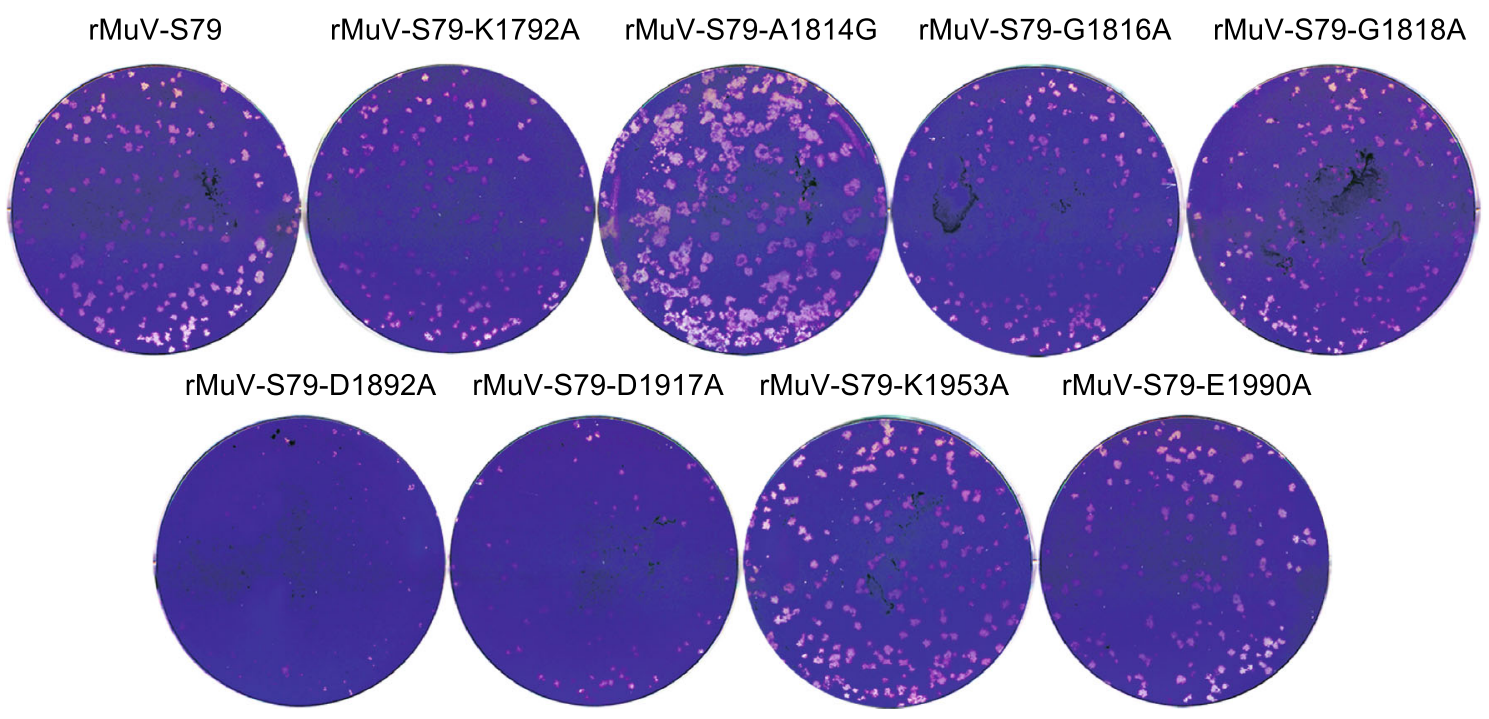

Fig. 3 Plaque morphology of rMuVs. A plaque assay was performed in Vero cells for each recombinant virus. After 7 days of incubation, plaques were fixed and stained with crystal violet. 

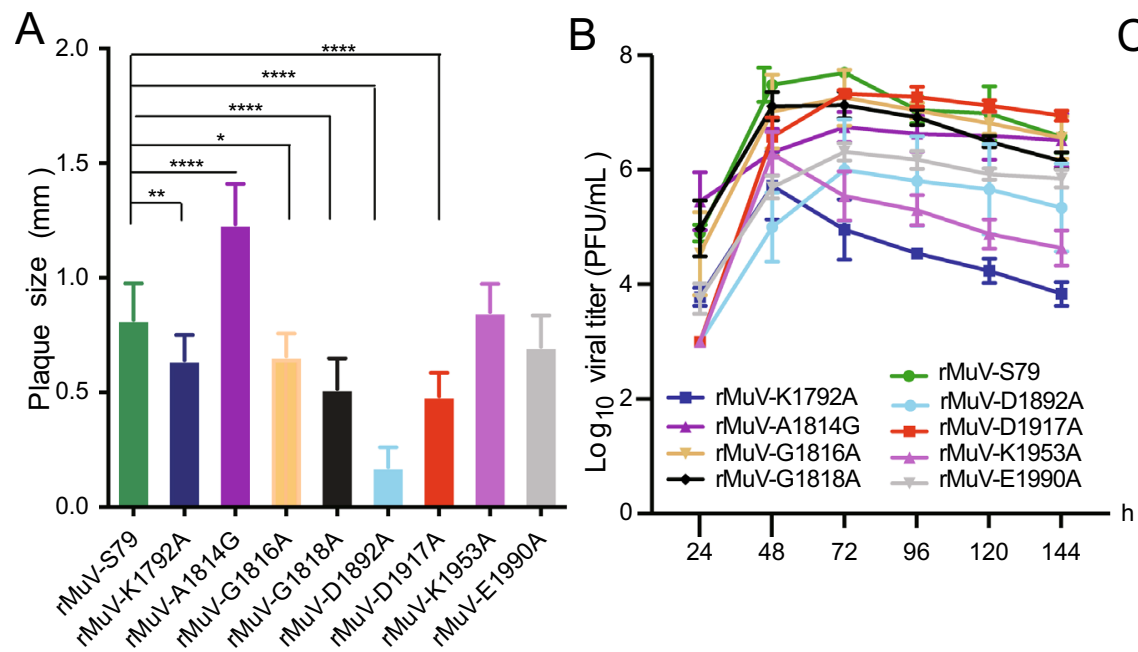

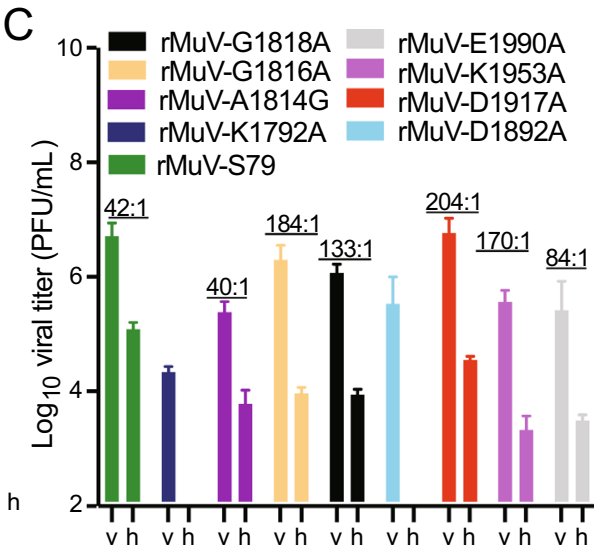

Fig. 4 Growth characteristics of rMuV mutants. A Comparison of plaque size of recombinant virus. Fifteen plaques of each recombinant virus were measured and the average plaque diameter was calculated. $* P<0.05 ; * * P<0.01, * * * P<0.001, * * * * P<0.0001$. B Growth kinetics of $\mathrm{rMuV}$ mutants. Vero cells were infected by each recombinant virus at an MOI of 0.1 , virus was harvested at the

$\left(7.6 \pm 0.22 \log _{10} \mathrm{PFU} / \mathrm{mL}\right)$. The rMuV-S79-K1953A and rMuV-S79-E1990A, with a similar plaque size as $\mathrm{rMuV}$ $\mathrm{S} 79$, had a lower peak titer $\left(5.55 \pm 0.43 \log _{10} \mathrm{PFU} / \mathrm{mL}\right.$ and $6.32 \pm 0.15 \log _{10} \mathrm{PFU} / \mathrm{mL}$ ) than that of the parental strain. The peak titer of rMuV-S79-D1917A was $7.33 \pm 0.06$ $\log _{10} \mathrm{PFU} / \mathrm{mL}$, which was comparable to the parental strain at $72 \mathrm{~h}$ after infection.

Next, we determined whether defects in rMuVs replication are cell type specific. Briefly, Vero (IFN-) and Hela $(\mathrm{IFN}+)$ cells were infected with $\mathrm{rMuVs}_{\mathrm{V}}$ and virus titers were determined in $72 \mathrm{~h}$ after infection by plaque assay. As shown in Fig. 4C, the ratio of viral titer between rMuV-S79 in Vero and Hela cells was 42:1. The ratio of viral titer between $\mathrm{rMuV}-\mathrm{S} 79-\mathrm{A} 1814 \mathrm{G}$ in Vero and Hela cells was 40:1 similar to the parental strain. The ratios of viral titer between rMuV-S79-G1816A, rMuV-S79-G1818A, rMuVS79-D1917A, rMuV-S79-K1953A and rMuV-S79-E1990A in Vero and Hela cells were 184:1, 133:1, 204:1, 170:1 and 84:1 respectively, which higher than that of the parental strain. rMuV-S79-K1792A and rMuV-S79-D1892A cannot be detected $>100 \mathrm{PFU} / \mathrm{mL}$ viral titer in Hela cells. These data indicated that the replication kinetics of $\mathrm{rMuVs}_{\mathrm{s}}$ in Hela cells was reduced and the reduction of $\mathrm{rMuVs}$ was more obvious than the parent strain.

The parental $\mathrm{rMuV}-\mathrm{S} 79$ induced significant $\mathrm{CPE}$ in Vero cells at $72 \mathrm{~h}$ post-inoculation (hpi). All rMuV-S79 mutants except rMuV-S79-A1814G had significantly delayed CPE comparing with the rMuV-S79 (Fig. 5). Recombinant rMuV-S79-A1814G developed an earlier $\mathrm{CPE}$, formed large syncytia at $24 \mathrm{hpi}$ and reached a maximum CPE at 48 hpi. These data showed that rMuV-S79- indicated time points. Virus titers of the rMuV mutants were determined by multiple step growth curve plaque assay in Vero cells. C Replication of rMuVs in IFN-/IFN + cell lines. Confluent Vero (v) and Hela cells (h) were infected with rMuVs at an MOI of 0.1 . After $72 \mathrm{~h}$ post infection, virus titers were determined by plaque assay.

A1814G had an increased syncytial formation whereas all other rMuV-S79 mutants had a significant attenuation in viral replication compared to the parental rMuV-S79.

\section{Genetic Stability of Recombinant rMuV-S79 Mutants in Cell Culture}

We then tested the genetic stability of these recombinant viruses in cell culture. All rMuV-S79 mutants were passaged 10 times in Vero cells. At each passage, the MTase region in the $L$ gene was amplified by RT-PCR and sequenced. The results showed that the $L$ gene in each passage retained the desired mutation. At passage 10, the entire genome of each rMuV-S79 mutant was sequenced. All viruses retained the desired mutations and no other mutations were found in the viral genome as showed in Supplementary Fig. S3. The mumps vaccines are produced in primary chicken embryo fibroblast cell (CEF), so we tested the genetic stability of these rMuVs in CEF cells. rMuVs carrying mutations were repeatedly passaged 3 times in CEF cell and the entire genome of each recombinant virus was amplified by RT-PCR and sequenced. No mutations were found in the genome. These results indicated that these rMuV-S79 mutants were genetically stable in cell culture.

\section{Replication of rMuV-S79 Mutants in Cotton Rats}

We next tested the replication ability of these $\mathrm{rMuV}$ mutants in vivo. Briefly, five-week-old, specific-pathogenfree (SPF) cotton rats were anesthetized with sevoflurane 


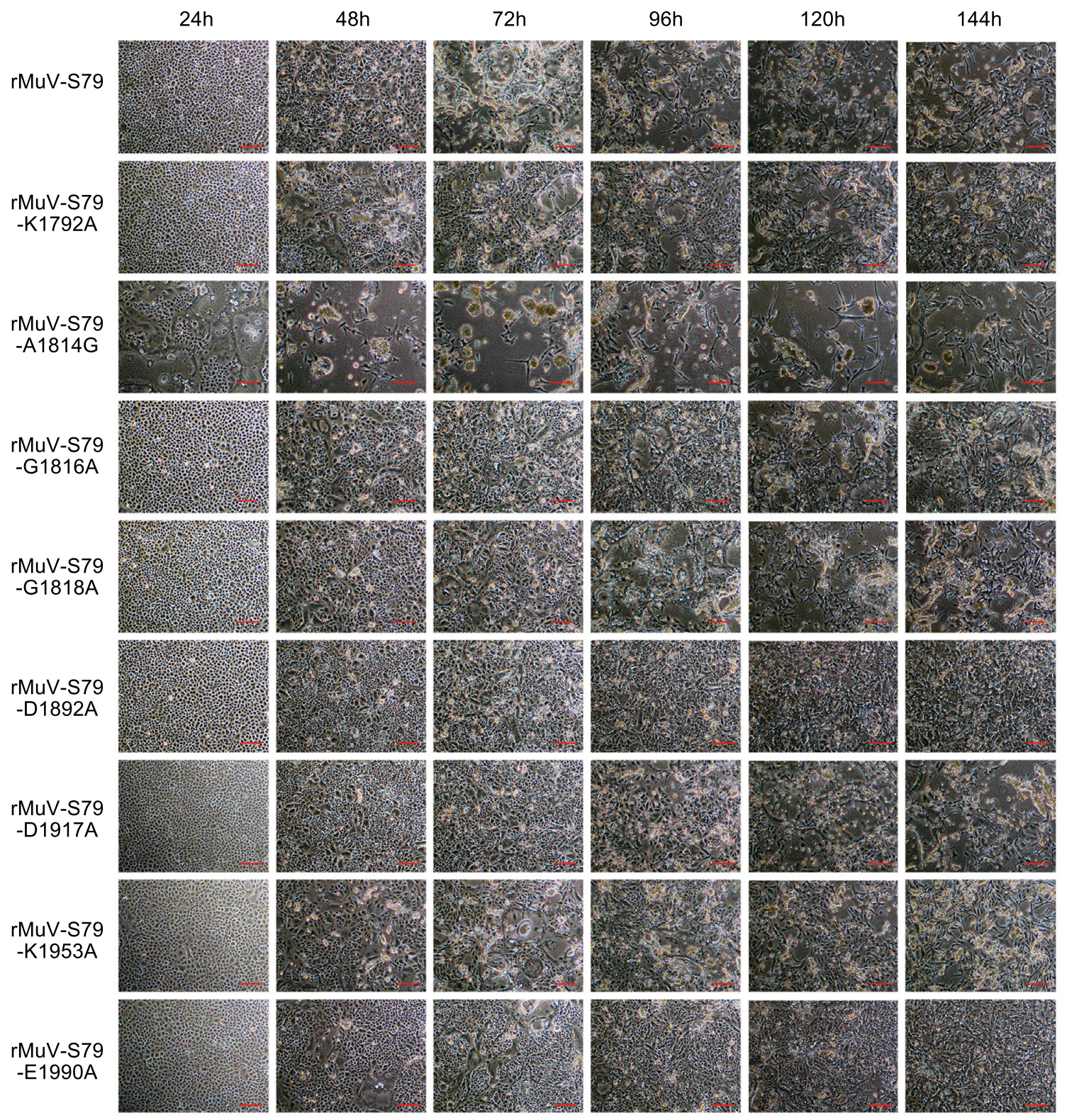

Fig. 5 Cytopathic effects (CPE) of recombinant MuVs carrying mutations. Vero cells were infected by each recombinant rMuV at an MOI of 0.1 . CPE was observed daily and photographed at 24, 48, 72, 96, 120 and $144 \mathrm{~h}$ after infection. Scale bar: $200 \mu \mathrm{m}$.

and inoculated intranasally with $1 \times 10^{6} \mathrm{PFU}$ of each rMuV-S79 mutant (except rMuV-S79-A1814G) or the parental rMuV-S79. No weight loss, death or obvious clinical signs of respiratory infection was observed in cotton rats. At day 4 post-inoculation, cotton rats were euthanized, lung, spleen and brain tissues were collected for virus titration and histology. No infectious $\mathrm{MuV}$ was detected in spleen and brain tissues for all groups. Viral titer in the lung tissues of rMuV-S79-G1818A-infected cotton rats was $2.97 \pm 0.15 \log _{10} \mathrm{PFU} / \mathrm{g}(P>0.05)$, which is similar to rMuV-S79-infected control group $\left(2.75 \pm 0.12 \log _{10} \mathrm{PFU} / \mathrm{g}\right)$. Viral titer in the rMuV-S79-
G1816A $\left(2.25 \pm 0.06 \log _{10}\right.$ PFU/g,$\left.P<0.001\right)$ and $\mathrm{rMuV}-$ S79-E1990A groups $\left(2.48 \pm 0.10 \log _{10} \mathrm{PFU} / \mathrm{g}, P<0.05\right)$ were significantly lower than that in the $\mathrm{rMuV}-\mathrm{S} 79$ group. However, no infectious virus was detected in the lungs of rMuV-S79-K1792A, rMuV-S79-D1892A and rMuV-S79K1953A groups (Table 1). Also, no significant histologic lesion was detected in the lung and brain tissues for all groups (Supplementary Fig.S4A, S6A). To determine the viral antigen distribution in lung and brain tissues, IHC was performed using an antibody against the $\mathrm{MuV} \mathrm{N}$ protein. As shown in Fig. 6, viral antigen-positive cells were detected inside the alveolar cells in lungs of rMuV-S79- 
infected cotton rats. Interestingly, the virus antigen-positive cells were detected in the bronchiolar cells of the $\mathrm{rMuV}$ K1792A, rMuV-D1892A and rMuV-E1990A infected group, which had lower viral replication kinetics. Fewer antigen-positive cells were detected at the alveolar cells in lung tissues of other rMuVs infected groups. No viral antigen was detected in brain tissues from all groups as shown in Supplementary Fig. S5A. These results showed that rMuV mutants were more attenuated in replication in cotton rats compared to the rMuV-S79 virus strain.

\section{rMuV-S79 Mutants Provided Complete Protection Against MuV Challenge}

Next, the immunogenicity of rMuV-S79 mutants was determined in cotton rats. Five-week-old SPF cotton rats were anesthetized with sevoflurane and inoculated intra-
Fig. 6 Immunohistochemical (IHC) staining of lungs of cotton rats infected with $\mathrm{rMuV}$ mutants. Cotton rats were intranasally inoculated with $1.0 \times 10^{6} \mathrm{PFU}$ of each $\mathrm{rMuV}$ mutant and were sacrificed at day 4 post-inoculation. The right lung from each cotton rat $(\mathrm{N}=45)$ was fixed with $4 \%$ formaldehyde and embedded in paraffin, sectioned at $4 \mu \mathrm{m}$ and stained with monoclonal antibody against mumps $\mathrm{N}$ protein (Abcam, ab9880, 1/500 dilution in PBS) to determine the distribution of viral antigen. Arrows, antigen-positive cells. Scale bar: $50 \mu \mathrm{m}$.
Table 1 Infectivity of rMuV mutants in cotton rats.
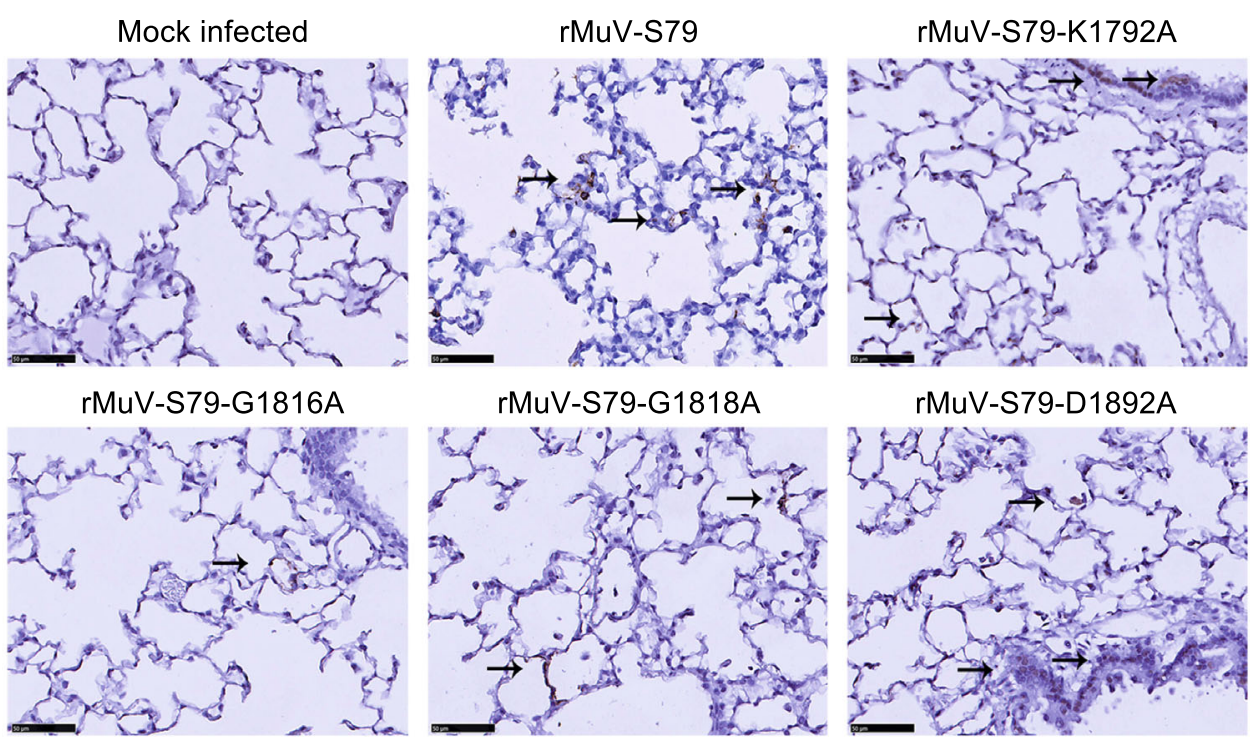

rMuV-S79-G1818A

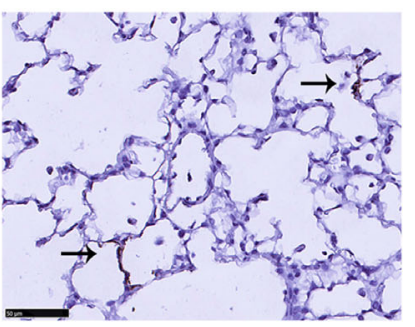

rMuV-S79-D1892A

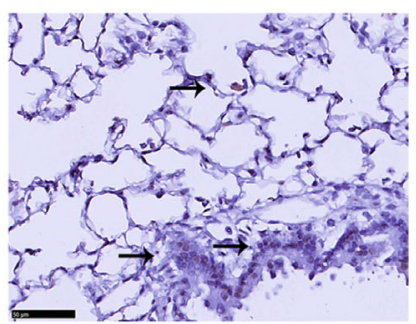

rMuV-S79-D1917A
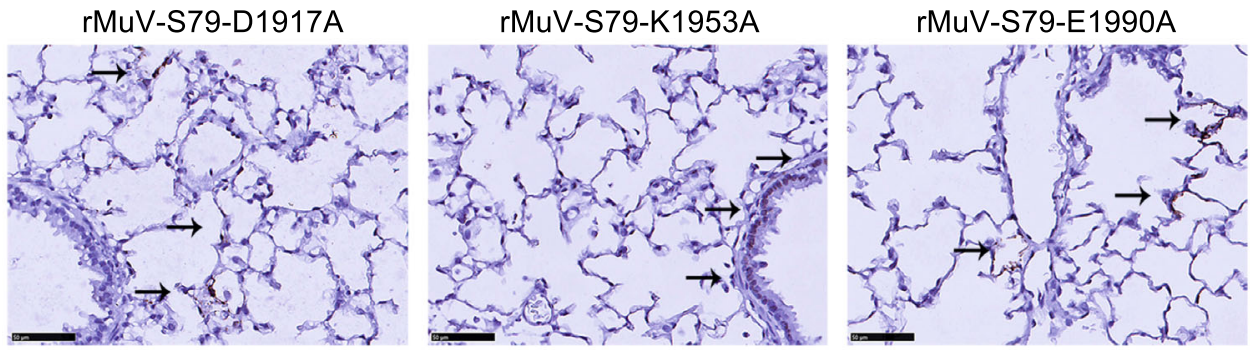

\begin{tabular}{lll}
\hline Group & \multicolumn{2}{l}{ Viral titer in lung } \\
\cline { 2 - 3 } & Percentage of infection & Viral titer $\left(\log _{10}\right.$ PFU/g) \\
\hline rMuV-S79 & 80 & $2.75 \pm 0.12$ \\
rMuV-S79-K1792A & 0 & $\mathrm{ND}$ \\
rMuV-S79-G1816A & 100 & $2.25 \pm 0.06$ \\
rMuV-S79-G1818A & 80 & $2.97 \pm 0.15$ \\
rMuV-S79-D1892A & 0 & $\mathrm{ND}$ \\
rMuV-S79-D1917A & 80 & $3.04 \pm 0.10$ \\
rMuV-S79-K1953A & 0 & $\mathrm{ND}$ \\
rMuV-S79-E1990A & 100 & $2.48 \pm 0.10$ \\
DMEM & 0 & $\mathrm{ND}$ \\
\hline
\end{tabular}

ND: not detected; Data each group were the mean of 5 cotton rats \pm SD 
nasally with $1 \times 10^{6} \mathrm{PFU}$ of each rMuV-S79 mutant or parental $\mathrm{rMuV}-\mathrm{S} 79$ and serum samples were collected weekly for determination of neutralizing antibody titer. At week 4 post-vaccination, each group was challenged with $1.0 \times 10^{7} \mathrm{PFU}$ of a wild type MuV strain. At 4 days postchallenge (dpc), cotton rats were euthanized; and lung tissues were collected for virus titration and histologic examination.

We compared the dynamics of neutralizing antibody responses after vaccination with each $\mathrm{rMuV}$ mutant. Figure 7A shows cotton rats vaccinated with the parental vaccine strain rMuV-S79 induced neutralizing antibodies at week 2 post-vaccination, reached a peak titer at week 3 and started to decline at week 4 . Antibody titers in rMuVS79-K1792A, rMuV-S79-D1892A, rMuV-S79-G1818A and $\mathrm{rMuV}-\mathrm{S} 79-\mathrm{K} 1953 \mathrm{~A}$ were not detectable at week 2, suggesting a significant delay in antibody responses for those mutants. Recombinant rMuV-S79-K1792A and
rMuV-S79-D1892A had significantly lower antibody titers than the rMuV-S79 group over the entire time period. Although rMuV-S79-G1818A and rMuV-S79-K1953A had a significant delay in antibody responses $(P<0.05)$ but reached a comparable peak titer with the rMuV-S79 group. Recombinant rMuV-S79-E1990A induced similar antibody responses to rMuV-S79 $(P>0.05)$. Importantly, neutralizing antibody titers in the rMuV-S79-D1917A group triggered significantly higher antibody titers in all time points comparing with the parental rMuV-S79 group $(P<0.05)$. Thus, these data demonstrated that rMuV-S79 mutants had variable impacts on antibody responses. Their ability of triggering neutralizing antibodies can be ranked as following: rMuV-S79-D1917A $>$ rMuV-S79, rMuVS79-E1990A > rMuV-S79-G1816A, rMuV-S79G1818A > rMuV-S79-K1953A > rMuV-S79-K1792A, rMuV-S79-D1892A.
A

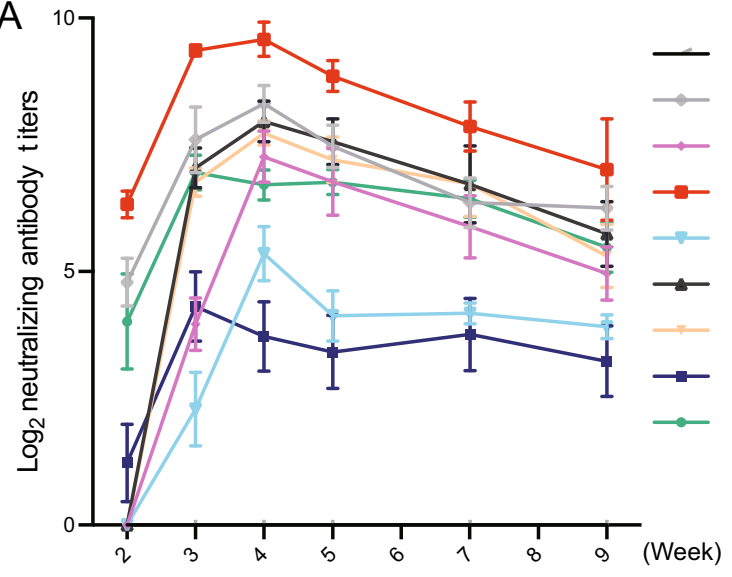

DMEM

rMuV-S79-E1990A

rMuV-S79-K1953A

rMuV-S79-D1917A

rMuV-S79-D1892A

rMuV-S79-G1818A

rMuV-S79-G1816A

rMuV-S79-K1792A

rMuV-S79

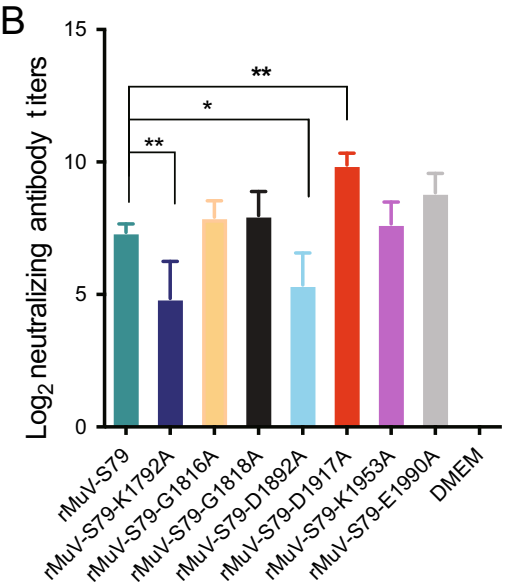

E

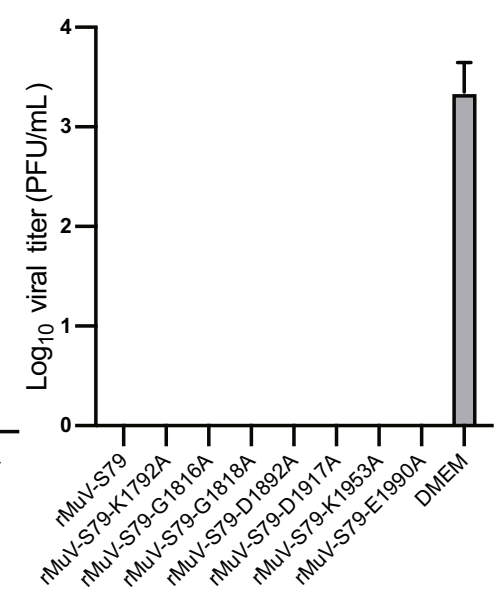

Fig. 7 Neutralizing antibody titers triggered by rMuVs in cotton rats. A Dynamics of neutralizing antibody titers triggered by each recombinant $\mathrm{MuV}$ in cotton rats. The serum of the cotton rats was collected at 2, 3, 4, 5, 7 and 9 weeks after immunization and antibody titers were determined using a plaque reduction neutralization assay. Data each group were the mean of 5 cotton rats \pm SEM.
B Comparison of the highest neutralizing antibody titers induced by each rMuVs in cotton rats. C Comparison of neutralizing antibodies at week 3. D Comparison of neutralizing antibodies at week 4 . E Protective ability of rMuV-S79 mutants in cotton rats. $* P<0.05 ; * * P<0.01 ; * * * P<0.001$. 
The peak antibody titer of the rMuV-S79-D1917 group was higher than that of the parental strain, however, the rMuV-S79-K1792A and rMuV-S79-D1892A groups had lower peak antibody titers than the parental strain; and the other groups were similar to the rMuV-S79 group (Fig. 7B). Importantly, neutralizing antibody titer in the rMuV-S79-D1917A group produced antibodies earlier and higher than that of the parental vaccine group $(P<0.05)$. We also analyzed the peak antibody titer either at week 3 or 4 post-immunization. At week 3, rMuV-S79-K1792A, rMuV-S79-D1892A and rMuV-S79-K1953A produced significantly lower neutralizing antibodies than the parental strain whereas rMuV-S79-D1917 produced a higher neutralizing antibody titer (Fig. 7C). At week 4, rMuV-S79D1892A and rMuV-S79-K1953A produced antibodies similar to the rMuV-S79, while rMuV-S79-D1917 and rMuV-S79-K1792A had the same trend as week 3 (Fig. 7D).

For the unvaccinated challenged control group, an average virus titer of $3.35 \pm 0.26 \log _{10} \mathrm{PFU} / \mathrm{g}$ was detected in the lungs (Fig. 7E). In contrast, no MuV titer was detected in the lungs from cotton rats vaccinated with each $\mathrm{rMuV}$ mutant and challenged with wild type MuV. These results showed that all $\mathrm{rMuV}-\mathrm{S} 79$ mutants provided complete protection against $\mathrm{MuV}$ replication in the lungs. No significant lung and brain histology lesion was found in all groups, even in the unvaccinated cotton rats (Supplementary Fig. S4B, S6B). A few of inflammatory cell infiltrations were found in the lung of cotton rats immunized with rMuVs.

For lung tissues from unvaccinated controls challenged with wild type $\mathrm{MuV}$, viral antigens were found inside the alveolar cells of lung tissues in all the cotton rats immunized with rMuVs (Fig. 8) and the viral antigens inside the alveolar cells in cotton rats vaccinated with rMuV-S79D1917A were fewest compared to others. A small amount of inflammatory cell infiltration could be observed around the antigen-positive cells, which may be related to virus clearance. No viral antigen was detected in brain tissues from all groups as shown in Supplementary Fig. S5B.

\section{Discussion}

A live attenuated vaccine (LAV) is one of the most promising vaccines for human paramyxoviruses and pneumoviruses. LAV has many advantages such as lack of enhanced lung diseases, ease of production, economically feasibility and induction of durable and strong immunity. In China, children receive MMR vaccine (measles, mumps and rubella) since 1990s and S79 mumps attenuated vaccine originating from $\mathrm{MuV} \mathrm{JL}$ strain was used for MMR vaccination (Pang et al. 2018). Although MMR vaccine is highly successful in controlling measles, mumps and rubella, mumps outbreaks in vaccinated populations have been reported in recent years (Cui et al. 2017, 2018; Ma C et al. 2018; Ma R et al. 2018). Therefore, developing a new $\mathrm{MuV}$ vaccine candidate with an improved safety and efficacy is still needed.

Traditional methods for preparing live attenuated mumps vaccines are time-consuming, high-costing and difficult to obtain stable mutations (Buynak and Hilleman 1966). The emerging reverse genetics technology allows us to modify the virus protein genes related to safety and immunogenicity and can obtain virus vaccine candidate strains in a short time, which is more controllable and rapid than traditional methods. Vero cell culture-based vaccine development allows producing high-dose vaccine in a more simplified way, surpassing the traditional technique of serial passage in chicken embryos. The traditional virus rescue method is based on T7 RNA polymerase driven by vaccinia virus, which inevitably suffers the problem of helper virus contamination. In this study, we used BHK cells stably expressing T7 RNA polymerase to rescue $\mathrm{rMuVs}$, which prevents the risk of being contaminated by vaccinia virus. This method has been approved in other studies for rescue of human metapneumovirus, bovine respiratory syncytial virus and measles virus (Ma et al. 2014; Sun et al. 2014; Zhang et al. 2014; Cui et al. 2017; Pang et al. 2018; Wang et al. 2018; Zhou et al. 2019b). We have effectively rescued rMuV-S79 without helper vaccinia virus in our previous researches (Zhou et al. 2019a, 2019b).

We aimed to increase the immunogenicity of the MuV vaccine and reduce its side effects by mutating the methyltransferase catalytic site and the SAM binding site of the L protein. We successfully rescued four rMuV-S79s carrying SAM binding site mutations ( $\mathrm{rMuV}-\mathrm{S} 79-\mathrm{A} 1814 \mathrm{G}$, rMuV-S79-G1816A, rMuV-S79-G1818A and rMuV-S79D1892A) and four mutants in MTase catalytic site (rMuVS79-K1792A，rMuV-S79-D1917A， rMuV-S79-K1953A and $\mathrm{rMuV}-\mathrm{S} 79-\mathrm{E} 1990 \mathrm{~A})$. Among these recombinant viruses, rMuV-S79-A1814G showed larger plaque size and faster progress of CPE than the parental virus. The SAM binding site in the L proteins of most NNS RNA viruses is an authentic GxGxG...D motif. However, the SAM binding site of $\mathrm{L}$ proteins of $\mathrm{MuV}, \mathrm{NDV}$ and PIV2 is an AxGxG...D motif, in which the first glycine residue is mutated to alanine. When the alanine is mutated back to glycine in SAM binding site of MuV L protein, the resultant recombinant virus can accelerate viral replication. Thus, the parental rMuV-S79 has a naturally occurring mutation in the SAM binding site, which may contribute to the attenuation of rMuV-S79. All other rMuV-S79 mutants were significantly more attenuated than the parental $\mathrm{rMuV}$ S79, which produced smaller plaque, delayed viral 
Fig. 8 Immunohistochemical (IHC) staining of lungs of cotton rats vaccinated with rMuVs followed by $\mathrm{MuV}$ challenge. Cotton rats were immunized intranasally with each $\mathrm{rMuV}$ mutant. At week 9 post-immunization, cotton rats were challenged with $1 \times 10^{7}$ $\mathrm{PFU}$ of a wild type $\mathrm{MuV}$ strain and were sacrificed at day 4 post-challenge. The right lung from each cotton rat $(\mathrm{N}=45)$ was fixed with $4 \%$

formaldehyde and embedded in paraffin, sectioned at $4 \mu \mathrm{m}$ and stained with monoclonal antibody against mumps $\mathrm{N}$ protein (Abcam, ab9880, 1/500 dilution in PBS) to determine the distribution of viral antigen. Scale bar: $50 \mu \mathrm{m}$
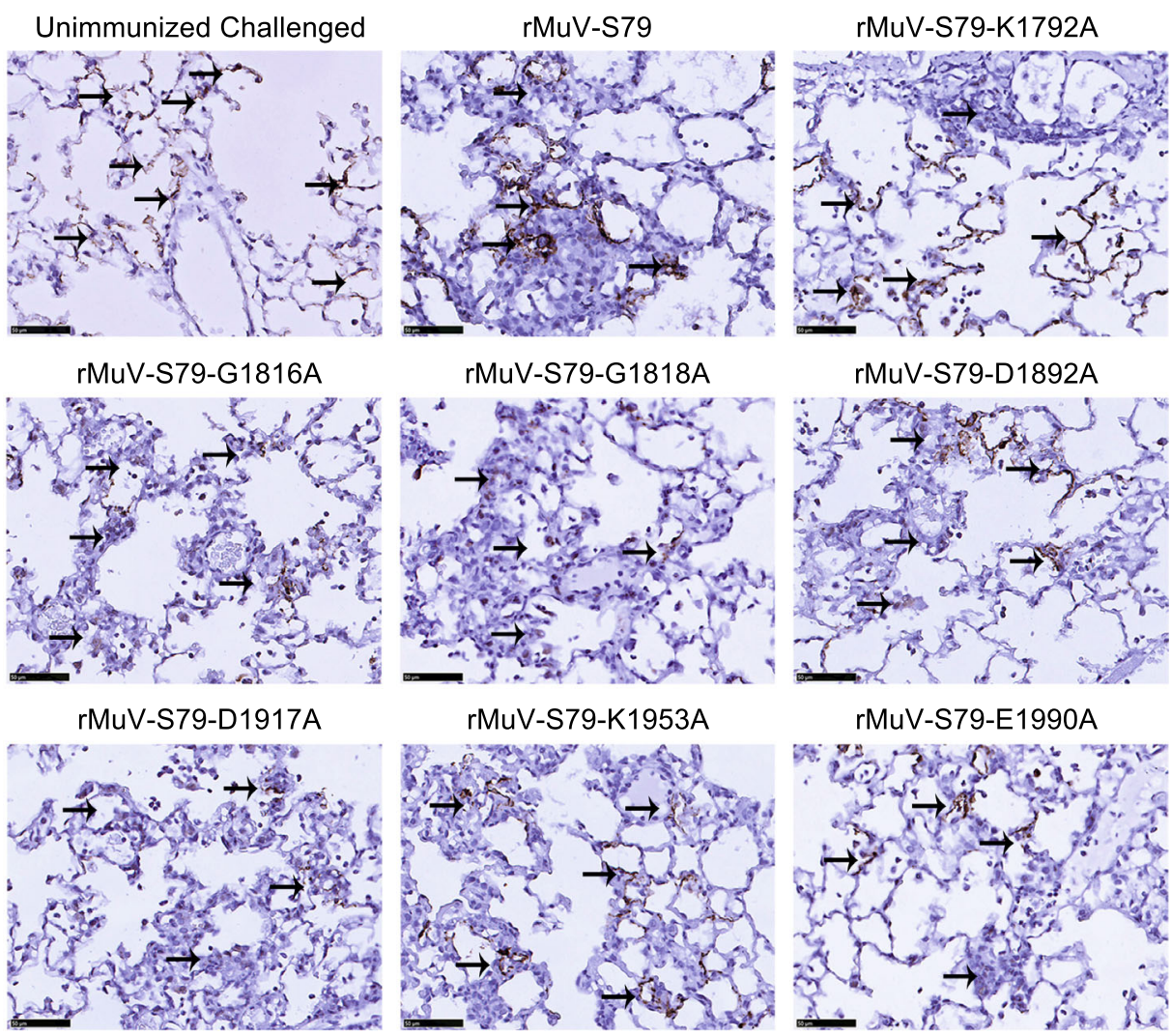

rMuV-S79-E1990A

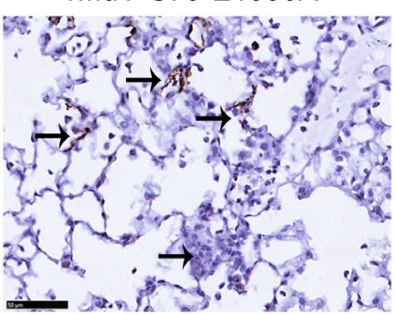

replication and delayed CPE progress. Most of $\mathrm{rMuV}$ mutants at the SAM binding site tend to affect the size of the virus plaque, while most of mutants at the catalytic site are more aggressive at affecting the virus titer. This demonstrates that mutations to the MTase catalytic site and SAM binding site further attenuated the $\mathrm{MuV}$, thereby enhancing the safety of the vaccine candidates.

Ferrets and monkeys are susceptible to MuV infection (Rubin et al. 1999; Parker et al. 2013), but their models are huge and expensive. For scientific research, the mouse animal model is a better experimental choice. However, mumps cannot be replicated well or cause disease in adult mice (Tsurudome et al. 1987; Cusi et al. 2001) and cannot be detected in the lungs of normal mice. At present, the newborn Lewis rat hydrocephalus model was used to evaluate the neurotoxicity of mumps vaccine by the severity of hydrocephalus by immunization of mumps virus in the brain (Wolinsky et al. 1976; Kristensson et al. 1984). Similar with the Jeryl Lynn mumps vaccine strain, the Chinese "S-79" mumps vaccine strain has been shown low neurotoxicity in monkey animal experiments ( $\mathrm{Su}$ et al. 2014). So, we did not use the newborn Lewis rat hydrocephalus model for neurotoxicity testing. Some researchers have used IFN- $\alpha / \beta \mathrm{R}^{-/-}$mice as an infectious model of mumps and found that IFN- $\alpha / \beta R^{-/-}$mice can be detected with a virus titer of $1000 \mathrm{PFU} / \mathrm{g}$ lung tissue in the lungs after mumps infection (Pickar et al. 2017), which was useful for evaluating the toxic reaction of mumps virus in mice. Recently, cotton rats have been regarded as improved small animal model for human paramyxoviruses and pneumoviruses including studying viral infection and pathogenesis, testing vaccine efficacy and evaluating antiviral drugs (Boukhvalova and Blanco 2013; Green et al. 2013). In our previous research, we found viruses can be detected in the lung of the cotton rat after mumps infection as in IFN- $\alpha / \beta \mathrm{R}^{-/-}$mice (Zhou et al. 2019b). In this study, we found that neither the parental rMuV-S79 nor wild type $\mathrm{MuV}$ strains caused significant histologic lesions in the lungs of unvaccinated cotton rats. However, both $\mathrm{MuV}$ strains replicated in the lungs of cotton rats and mumps viral antigens can be detected inside the alveolar cells of cotton rats. These results suggested that cotton rats might be a good model for viral replication and viral antigen detection in the lungs but not lung pathology. In cotton rats, rMuV-S79-D1917A and rMuV-S79-G1818A showed a similar replication capacity in the lungs and lower virus titer was found in rMuV-S79-G1816A and rMuV-S79-E1990A compared to rMuV-S79. rMuV-S79K1792A, rMuV-S79-D1892A and rMuV-S79-K1953A did not have detectable infectious virus in the lungs. These results demonstrated that most of these $\mathrm{rMuV}$ mutants were further attenuated in replication in vivo.

rMuV-S79-G1816A, rMuV-S79-G1818A and rMuVS79-E1990A groups produced neutralizing antibodies 
similar to the parent rMuV-S79. Importantly, rMuV-S79D1917A triggered higher neutralizing antibodies than the parent rMuV-S79 $(P<0.05)$, with fewest viral antigen detection provided complete protection against wild type $\mathrm{MuV}$ infection. This suggested that rMuV-S79-D1917A had a higher immunogenicity than rMuV-S79. These rMuVs will be ideal candidates for mumps vaccines. rMuV-S79-K1792A, rMuV-S79-D1892A and rMuV-S79K1953A are safer in vitro/vivo than the rMuV-S79 strain, but produces lower neutralizing antibodies, which were not suitable as candidates of mumps vaccine. It has been recognized that mRNA stability and efficient translation are related to G-N-7 methylation, while $2^{\prime}-\mathrm{O}$ methylation is important for distinguishing between self and non-self mRNA (Zust et al. 2011; Sun et al. 2014). Both the mutations in mRNA methyltransferase catalytic site and the SAM binding site may affect the level of G-N-7 methylation and/or ribose $2^{\prime}-\mathrm{O}$ methylation of mRNA. The replication kinetics of rMuVs in Hela cells showed that rMuV-S79-K1792A and rMuV-S79-K1892A were pronounced reduced than the parent strain. The 2'-O methylation functions of these two rMuVs may be affected, but it is uncertain whether there is a loss of G-N-7 methylation function. While rMuV-S79-A1814G and rMuV-S79E1990A may be less affected by interferon and may be more attenuated by G-N-7 methylation. For rMuV-S79D1917A, which had a higher immunogenicity than rMuVS79, we think the change of this site may only affect the catalytic activity of $2^{\prime}-\mathrm{O}$ methylation. The attenuated mechanism of $2^{\prime}-\mathrm{O}$ methylation virus enhances the sensitivity to IFN antiviral effects (Daffis et al. 2010) and can also promote the expression of interferon (Zust et al. 2011). Interferon can promote cellular immunity and humoral immunity, thereby affecting the expression level of antibodies. Limited by radioactive experimental conditions, we failed to detect the impact of the respective mutations on the G-N-7 or 2'-O methylation of mRNA methyltransferase. The methylation of mRNA methyltransferase of rMuVs needs to be confirmed by further researches.

Adult mice are insensitive to mumps infection, but newborn mice infected with mumps may cause significant lung histology lesion and even death. Immunizing maternal cotton rats as described, then allow these cotton rats to mate, the newborn cotton rats will obtain the $\operatorname{IgG}$ theoretically. Therefore, the neonatal cotton rats may be potential animal model to evaluate histopathology and the protection efficiency of the mumps vaccine. In this study, we only tested the B cell immune response of cotton rat mumps and did not evaluate the specific $\mathrm{T}$ cell immune response to the live attenuated vaccine. We will complete the evaluation of vaccine immunity in the further development of vaccines. In future research, we will conduct multiple mutations at multiple sites of mRNA methyltransferase to achieve our goal that increase the immunogenicity of the $\mathrm{MuV}$ vaccine and reduce its side effects.

Finally, the current mumps epidemic strain in China is type F (Wu et al. 1998) and the S-79 mumps vaccine is type A strain. The F genotype vaccine produced a higher neutralizing antibody titer against the $\mathrm{F}$ genotype strain than the A genotype (Jeryl Lynn) vaccine (Zengel et al. 2017). Besides, the attenuated live mumps vaccine of the $F$ genotype has been used to prevent mumps in China (Liang et al. 2010). The method of improving vaccine safety and immunogenicity in this study can also be applied to the attenuated live mumps vaccine of the $\mathrm{F}$ genotype. Furthermore, the mumps virus S79 vaccine strain could as a carrier to express antigenic proteins of $\mathrm{F}$ genotype mumps viruses for preventing mumps viral infections with the reverse genetics system in our future research.

Acknowledgements This work was supported by The National Natural Science Foundation for Young Scholars of China (81901679), The Natural Science Foundation for Young Scholars of Zhejiang Province (LQ19H100005) and China Postdoctoral Science Foundation (2019M662076).

Author contributions $\mathrm{ZZ}$ and $\mathrm{Y}-\mathrm{WH}$ designed and guided the experiments in this manuscriptstudy. $\mathrm{XH}$ and $\mathrm{YW}$ conducted the main work of the experiment, data analysis and article writing. RL and BW provided help with cell experiments, while $\mathrm{MZ}$ and $\mathrm{DZ}$ provided support from animal technicians. MZ made an article revision. $\mathrm{ZZ}$ and Y-WH finalized the article. All authors read and approved the final manuscript.

\section{Compliance with Ethical Standards}

Conflict of interest The authors declare that they have no competing interests.

Animal and Human Rights Statement This study was approved by the Ethical Committee of Children's Hospital, Zhejiang University School of Medicine (No. 2013119). Written informed consents were obtained from all patients. All animal procedures conformed to the Guide for the Care and Use of Laboratory Animals and were approved by the Zhejiang University Medical Laboratory Animal Care and Use Committee.

\section{References}

Albertson JP, Clegg WJ, Reid HD, Arbise BS, Pryde J, Vaid A, Thompson-Brown R, Echols F (2016) Mumps Outbreak at a University and Recommendation for a Third Dose of MeaslesMumps-Rubella Vaccine - Illinois, 2015-2016. MMWR Morb Mortal Wkly Rep 65:731-734

Beleni AI, Borgmann S (2018) Mumps in the vaccination age: global epidemiology and the situation in Germany. Int $\mathrm{J}$ Environ Res Public Health 15:1618

Boukhvalova MS, Blanco JC (2013) The cotton rat Sigmodon hispidus model of respiratory syncytial virus infection. Curr Top Microbiol Immunol 372:347-358 
Bukreyev A, Camargo E, Collins PL (1996) Recovery of infectious respiratory syncytial virus expressing an additional, foreign gene. J Virol 70:6634-6641

Buynak EB, Hilleman MR (1966) Live attenuated mumps virus vaccine. 1. Vaccine development. Proc Soc Exp Biol Med 123:768-775

Cardemil CV, Dahl RM, James L, Wannemuehler K, Gary HE, Shah M, Marin M, Riley J, Feikin DR, Patel M, Quinlisk P (2017) Effectiveness of a Third Dose of MMR Vaccine for Mumps Outbreak Control. N Engl J Med 377:947-956

Clarke DK, Sidhu MS, Johnson JE, Udem SA (2000) Rescue of mumps virus from cDNA. J Virol 74:4831-4838

Cui A, Zhu Z, Hu Y, Deng X, Sun Z, Zhang Y, Mao N, Xu S, Fang X, Gao H, Si Y, Lei Y, Zheng H, He J, Wu H, Xu W (2017) Mumps Epidemiology and Mumps Virus Genotypes Circulating in Mainland China during 2013-2015. PLoS ONE 12:e0169561

Cui A, Zhu Z, Mao N, Si Y, Ma Y, Hu Y, Deng X, Wang L, Zeng L, Zhang Y, Xu W (2018) Assessment of one-dose mumpscontaining vaccine effectiveness on wild-type genotype $\mathrm{F}$ mumps viruses circulating in mainland China. Vaccine 36:5725-5731

Cusi MG, Correale P, Valassina M, Sabatino M, Valensin PE, Donati M, Gluck R (2001) Comparative study of the immune response in mice immunized with four live attenuated strains of mumps virus by intranasal or intramuscular route. Arch Virol 146:1241-1248

Daffis S, Szretter KJ, Schriewer J, Li J, Youn S, Errett J, Lin TY, Schneller S, Zust R, Dong H, Thiel V, Sen GC, Fensterl V, Klimstra WB, Pierson TC, Buller RM, Gale M Jr, Shi PY, Diamond MS (2010) 2'-O methylation of the viral mRNA cap evades host restriction by IFIT family members. Nature 468:452-456

Di Pietrantonj C, Rivetti A, Marchione P, Debalini MG, Demicheli V (2020) Vaccines for measles, mumps, rubella and varicella in children. Cochrane Database Syst Rev 4:004407

Elango N, Varsanyi TM, Kovamees J, Norrby E (1988) Molecular cloning and characterization of six genes, determination of gene order and intergenic sequences and leader sequence of mumps virus. J Gen Virol 69:2893-2900

Elliott GD, Yeo RP, Afzal MA, Simpson EJ, Curran JA, Rima BK (1990) Strain-variable editing during transcription of the $P$ gene of mumps virus may lead to the generation of non-structural proteins NS1 (V) and NS2. J Gen Virol 71:1555-1560

Fields VS, Safi H, Waters C, Dillaha J, Capelle L, Riklon S, Wheeler JG, Haselow DT (2019) Mumps in a highly vaccinated Marshallese community in Arkansas, USA: an outbreak report. Lancet Infect Dis 19:185-192

Galazka AM, Robertson SE, Kraigher A (1999) Mumps and mumps vaccine: a global review. Bull World Health Organ 77:3-14

Garcin D, Pelet T, Calain P, Roux L, Curran J, Kolakofsky D (1995) A highly recombinogenic system for the recovery of infectious Sendai paramyxovirus from cDNA: generation of a novel copyback nondefective interfering virus. EMBO J 14:6087-6094

Ge P, Tsao J, Schein S, Green TJ, Luo M, Zhou ZH (2010) Cryo-EM model of the bullet-shaped vesicular stomatitis virus. Science 327:689-693

Gilman MSA, Liu C, Fung A, Behera I, Jordan P, Rigaux P, Ysebaert N, Tcherniuk S, Sourimant J, Eleouet JF, Sutto-Ortiz P, Decroly E, Roymans D, Jin Z, McLellan JS (2019) Structure of the respiratory syncytial virus polymerase complex. Cell 179:193-204

Green MG, Huey D, Niewiesk S (2013) The cotton rat (Sigmodon hispidus) as an animal model for respiratory tract infections with human pathogens. Lab Anim (NY) 42:170-176

Jin H, Clarke D, Zhou HZ, Cheng X, Coelingh K, Bryant M, Li S (1998) Recombinant human respiratory syncytial virus (RSV) from cDNA and construction of subgroup A and B chimeric RSV. Virology 251:206-214

Kristensson K, Orvell C, Malm G, Norrby E (1984) Mumps virus infection of the developing mouse brain-appearance of structural virus proteins demonstrated with monoclonal antibodies. J Neuropathol Exp Neurol 43:131-140

Lawson ND, Stillman EA, Whitt MA, Rose JK (1995) Recombinant vesicular stomatitis viruses from DNA. Proc Natl Acad Sci U S A 92:4477-4481

Lewnard JA, Grad YH (2018) Vaccine waning and mumps reemergence in the United States. Sci Transl Med 10:5945

Li J, Wang JT, Whelan SP (2006) A unique strategy for mRNA cap methylation used by vesicular stomatitis virus. Proc Natl Acad Sci U S A 103:8493-8498

Liang Y, Ma S, Liu L, Zhao H, Wang L, Jiang L, Xie Z, Dong C, Li Q (2010) Identification and development of a promising novel mumps vaccine candidate strain. Microbes Infect 12:1178-1187

Ma C, Liu Y, Tang J, Jia H, Qin W, Su Y, Wang H, Hao L (2018) Assessment of mumps-containing vaccine effectiveness during an outbreak: Importance to introduce the 2-dose schedule for China. Hum Vaccin Immunother 14:1392-1397

Ma R, Lu L, Zhou T, Pan J, Chen M, Pang X (2018) Mumps disease in Beijing in the era of two-dose vaccination policy, 2005-2016. Vaccine 36:2589-2595

Ma Y, Wei Y, Zhang X, Zhang Y, Cai H, Zhu Y, Shilo K, Oglesbee M, Krakowka S, Whelan SP, Li J (2014) mRNA cap methylation influences pathogenesis of vesicular stomatitis virus in vivo. J Virol 88:2913-2926

Marin M, Marlow M, Moore KL, Patel M (2018) Recommendation of the Advisory Committee on Immunization Practices for Use of a Third Dose of Mumps Virus-Containing Vaccine in Persons at Increased Risk for Mumps During an Outbreak. MMWR Morb Mortal Wkly Rep 67:33-38

May M, Rieder CA, Rowe RJ (2018) Emergent lineages of mumps virus suggest the need for a polyvalent vaccine. Int J Infect Dis $66: 1-4$

Muhlemann K (2004) The molecular epidemiology of mumps virus. Infect Genet Evol 4:215-219

Muthukrishnan S, Morgan M, Banerjee AK, Shatkin AJ (1976) Influence of 5'-terminal $\mathrm{m} 7 \mathrm{G}$ and 2'-O-methylated residues on messenger ribonucleic acid binding to ribosomes. Biochemistry 15:5761-5768

Nogales A, Martinez-Sobrido L (2016) Reverse genetics approaches for the development of influenza vaccines. Int J Mol Sci 18:20

Pang H, Zhou Y, Zhao W, Jiang Q (2018) Seroprevalence and determinants associated with mumps antibodies after 20 Years of MMR vaccination in urban area of Shanghai. Int J Environ Res Public Health 15:2089

Parker L, Gilliland SM, Minor P, Schepelmann S (2013) Assessment of the ferret as an in vivo model for mumps virus infection. J Gen Virol 94:1200-1205

Pickar A, Xu P, Elson A, Zengel J, Sauder C, Rubin S, He B (2017) Establishing a small animal model for evaluating protective immunity against mumps virus. PLoS ONE 12:e0174444

Poch O, Blumberg BM, Bougueleret L, Tordo N (1990) Sequence comparison of five polymerases (L proteins) of unsegmented negative-strand RNA viruses: theoretical assignment of functional domains. J Gen Virol 71:1153-1162

Rubin S, Eckhaus M, Rennick LJ, Bamford CG, Duprex WP (2015) Molecular biology, pathogenesis and pathology of mumps virus. J Pathol 235:242-252

Rubin SA, Snoy PJ, Wright KE, Brown EG, Reeve P, Beeler JA, Carbone KM (1999) The mumps virus neurovirulence safety test in Rhesus monkeys: a comparison of mumps virus strains. J Infect Dis 180:521-525 
Saika S, Kidokoro M, Kubonoya H, Ito K, Ohkawa T, Aoki A, Nagata N, Suzuki K (2006) Development and biological properties of a new live attenuated mumps vaccine. Comp Immunol Microbiol Infect Dis 29:89-99

Su M, Mao Y, Shi TC, Liao W, Xu XF (2014) Evaluation of the neurovirulence of the strain s79 working seed lot of live attenuated mumps vaccines in rhesus monkeys. Chinese Journal of New Drugs 23:1267-1272

Sun J, Wei Y, Rauf A, Zhang Y, Ma Y, Zhang X, Shilo K, Yu Q, Saif YM, Lu X, Yu L, Li J (2014) Methyltransferase-defective avian metapneumovirus vaccines provide complete protection against challenge with the homologous Colorado strain and the heterologous Minnesota strain. J Virol 88:12348-12363

Tsurudome M, Yamada A, Hishiyama M, Ito Y (1987) Replication of mumps virus in mouse: transient replication in lung and potential of systemic infection. Arch Virol 97:167-179

Wang Y, Liu R, Lu M, Yang Y, Zhou D, Hao X, Zhou D, Wang B, Li J, Huang YW, Zhao Z (2018) Enhancement of safety and immunogenicity of the Chinese Hu191 measles virus vaccine by alteration of the S-adenosylmethionine (SAM) binding site in the large polymerase protein. Virology 518:210-220

Westphal DW, Eastwood A, Levy A, Davies J, Huppatz C, Gilles M, Lyttle H, Williams SA, Dowse GK (2019) A protracted mumps outbreak in Western Australia despite high vaccine coverage: a population-based surveillance study. Lancet Infect Dis 19:177-184

Whelan SP, Barr JN, Wertz GW (2004) Transcription and replication of nonsegmented negative-strand RNA viruses. Curr Top Microbiol Immunol 283:61-119

Wolinsky JS, Klassen T, Baringer JR (1976) Persistence of neuroadapted mumps virus in brains of newborn hamsters after intraperitoneal inoculation. J Infect Dis 133:260-267

Wu L, Bai Z, Li Y, Rima BK, Afzal MA (1998) Wild type mumps viruses circulating in China establish a new genotype. Vaccine $16: 281-285$
Wu L, Hu J, Li Z, Yang J, Sun X (2018) Epidemiology of mumps in Shanghai, 1990-2015. Chin J Vaccines Immun 024:43-47

Xu P, Chen Z, Phan S, Pickar A, He B (2014) Immunogenicity of novel mumps vaccine candidates generated by genetic modification. J Virol 88:2600-2610

Xu P, Li Z, Sun D, Lin Y, Wu J, Rota PA, He B (2011) Rescue of wild-type mumps virus from a strain associated with recent outbreaks helps to define the role of the SH ORF in the pathogenesis of mumps virus. Virology 417:126-136

Yang YT, Chow YH, Hsiao KN, Hu KC, Chiang JR, Wu SC, Chong P, Liu CC (2016) Development of a full-length cDNA-derived enterovirus A71 vaccine candidate using reverse genetics technology. Antiviral Res 132:225-232

Zengel J, Phan SI, Pickar A, Xu P, He B (2017) Immunogenicity of mumps virus vaccine candidates matching circulating genotypes in the United States and China. Vaccine 35:3988-3994

Zhang Y, Wei Y, Zhang X, Cai H, Niewiesk S, Li J (2014) Rational design of human metapneumovirus live attenuated vaccine candidates by inhibiting viral mRNA cap methyltransferase. J Virol 88:11411-11429

Zhou D, Zhu MY, Wang YL, Hao XQ, Zhou DM, Liu RX, Zhang CD, Qu CF, Zhao ZY (2019a) Attenuated MuV-S79 as vector stably expressing foreign gene. World J Pediatr 15:511-515

Zhou D, Zhu MY, Wang YL, Hao XQ, Zhou DM, Liu RX, Zhang CD, Qu CF, Zhao ZY (2019b) Establishment of an efficient reverse genetic system of Mumps virus S79 from cloned DNA. World J Pediatr 15:499-505

Zust R, Cervantes-Barragan L, Habjan M, Maier R, Neuman BW, Ziebuhr J, Szretter KJ, Baker SC, Barchet W, Diamond MS, Siddell SG, Ludewig B, Thiel V (2011) Ribose 2'-O-methylation provides a molecular signature for the distinction of self and non-self mRNA dependent on the RNA sensor Mda5. Nat Immunol 12:137-143 